\title{
L’avenir de la socioéconomie écologique. Environnement et valeur au-delà du Yalta disciplinaire
}

\author{
Gildas Renou*
}

Science politique, Université de Lorraine, Institut de recherches sur l'évolution de la nation et de l'État, Nancy, France

\begin{abstract}
Résumé - Dans leur récent ouvrage, les économistes Ali Douai et Gaël Plumecocq considèrent que l'absence de théorie de la valeur constitue une lacune majeure, tant scientifique que politique, de la socioéconomie écologique actuelle. Cet article exprime son désaccord avec cette thèse et en expose les raisons. Il suggère au contraire que le champ interdisciplinaire de la socioéconomie écologique s'abstienne de toute définition a priori de la valeur, afin de rendre les chercheurs attentifs aux différentes façons d'attribuer une valeur à l'environnement et aux entités naturelles. Pour rendre compte de l'intérêt de ce déplacement, l'article s'attache à présenter des outils permettant d'étudier les processus sociaux d'attribution de valeur à l'environnement. Deux approches - en apparence éloignées - s'avèrent complémentaires: le courant « conventionnaliste » en sociologie et, en économie, l' « environnementalisme des pauvres » de Joan MartinezAlier.
\end{abstract}

Mots-clés : valeur(s) / socioéconomie écologique / interdisciplinarité / sociologie des conventions / théorie marxiste / environnementalisme des pauvres / pluralisme en sciences sociales

\begin{abstract}
The future of ecological socioeconomics. Environment and value beyond academic boundaries. In their recent book, economists Ali Douai and Gaël Plumecocq consider the absence of value theory as a major lack, both political and scientific, of current ecological social economics. The author disagrees with this position and explains the reasons why. He suggests to found the interdisciplinary conversation in "ecological social economics" on the absence of any a priori definition of value, to enable researchers to fully take into account the plurality of ways people attribute a value to their environment or to natural entities. In order to illustrate the interest of this methodological twist, the article highlights two complementary approaches: the "conventionalist" stream in sociological economics and the work of Joan Martinez-Alier on the environmentalism of the Poor. They provide precious tools to help researchers study the social processes of valuing nature and the environment.
\end{abstract}

Keywords: value(s) / ecological socioeconomics / interdisciplinarity / sociology of conventions / Marxist theory / environmentalism of the Poor / pluralism in social sciences

La publication en langue française de ce que l'on peut considérer comme le premier manuel consacré à l'économie écologique (L'économie écologique, Douai et Plumecocq, La Découverte, 2017) constitue une excellente nouvelle pour tout le champ des « humanités environnementales » (Choné et al., 2016; Blanc et al., 2017). Il existait jusqu'à présent des articles synthétiques consacrés à l'économie écologique (Froger et al., 2016; Boisvert, 2017), mais pas

\footnotetext{
*Auteur correspondant :

gildas.renou@univ-lorraine.fr
}

d'ouvrage complet à vocation pédagogique. La façon dont les économistes Ali Douai et Gaël Plumecocq se sont acquittés de cette gageure redouble la bonne nouvelle. Leur ouvrage propose, en effet, au lecteur un atlas très précis d'un vaste paysage intellectuel en évolution constante, mais parfois difficilement accessible au lecteur francophone.

Ce livre se double d'un plaidoyer en faveur de l'une des principales perspectives intellectuelles en débat au sein de ce champ de recherche : la socioéconomie écologique, notamment promue par Clive Spash (2011). Plutôt que d'enfermer cette approche dans la seule discipline des 
sciences économiques, les auteurs ont l'ambition de participer à la constitution d'une perspective dans laquelle les diverses sciences humaines et sociales contribueraient de façon interdisciplinaire au renouvellement des sciences de l'oikos (la «maison commune»), en faisant enfin discuter les traditions écologiques, sociologiques et économiques, trop longtemps séparées. Douai et Plumecocq entendent ainsi faire de la socioéconomie écologique une double alternative. D'une part, une alternative à l'hégémonie de l'analyse économique standard étendue aux enjeux écologiques (Froger et al., 2016, p. 8), dont la marque sur la conception des dispositifs de régulation est devenue préoccupante (marché du carbone, rétribution de «services écosystémiques», etc.). D'autre part, une alternative aux thématiques interdisciplinaires en vogue, conceptuellement insatisfaisantes aux yeux de nos auteurs, de «l'Anthropocène» aux sciences de la durabilité («sustainability science»). La socioéconomie écologique devrait permettre, selon eux, de « repolitiser l'histoire et les enjeux environnementaux", afin de donner une place centrale à la prise en compte des « structures sociales et des institutions qui régissent nos rapports à la nature» (p. 10). C'est pourquoi ils regrettent que, telle qu'elle existe aujourd'hui, la socioéconomie écologique «ne s'est pas dotée d'une théorie de la valeur et a laissé dans ce domaine le champ libre à l'économie standard » (p. 99).

Notre propos se bornera à la discussion de ce seul aspect. Il laissera donc de côté l'immense majorité des développements de cet excellent ouvrage ${ }^{1}$. La version de la socioéconomie écologique que les auteurs promeuvent nous apparait, en effet, appeler une discussion sur un point précis : le fait qu'elle conditionne la compréhension et la contestation des destructions de la nature et des injustices socioenvironnementales à un préalable : l'adoption d'une position théorique, issue de la critique marxienne de la valeur. Celle-ci, on s'en souvient, est exposée dans le Livre I du Capital, à partir de l'étude des liens entre marchandise, monnaie et force de travail. Selon nos auteurs, l'impératif de repolitisation des enjeux environnementaux par la socioéconomie écologique impliquerait de partir d'un fondement assuré : la théorie de la valeur assise sur les catégories de la critique scientifique de l'exploitation capitaliste élaborée par Karl Marx. Ce réquisit, apparemment légitime, est-il aussi souhaitable que nos auteurs le laissent penser? Est-il pertinent de confier à une seule science la responsabilité de «repolitiser» les questions socioenvironnementales? Quels peuvent être les effets de ce postulat sur la coopération entre sciences économiques et sciences sociales et politiques?

\footnotetext{
${ }^{1}$ Voir aussi, dans ce même numéro, le texte d'Olivier Petit, qui propose une lecture croisée de trois ouvrages récents relevant de la socioéconomie écologique, dont celui de Douai et Plumecocq.
}

\section{La valeur, entre science et politique}

Il n'est ici pas question de remettre en cause la pertinence actuelle, tant intellectuelle que politique, de la critique marxienne de la valeur, ni même les remarquables tentatives de mises à jour de ce cadre analytique à la lumière des transformations contemporaines du capitalisme, qu'il s'agisse de la financiarisation, de l'augmentation des inégalités sociales ou de la crise écologique globale (Foster et al., 2010; Tanuro, 2010; Harribey, 2015). Nous souhaitons seulement contribuer à inscrire le dialogue interdisciplinaire au sein de la socioéconomie écologique sur un terrain intellectuellement fertile, reposant sur un équilibre et un respect de la diversité des contributions disciplinaires. L'argument défendu ici est qu'une socioéconomie écologique accueillante à une pluralité d'apports appelle un déplacement méthodologique qui pourra apparaître coûteux à beaucoup. Mais il nous semble constituer une garantie minimale d'un dialogue entre les économistes et les autres praticiens (sociologues, géographes, historiens, etc.), qui permette une intercompréhension et une complémentarité des apports disciplinaires.

Le déplacement suggéré consiste à accepter explicitement de ne pas se donner a priori de théorie économique de la valeur, afin de saisir et d'étudier la pluralité des dynamiques sociales et politiques d'attribution de valeur, en situation. Contrairement à ce qu'avancent nos auteurs, cette ouverture ne constitue ni une lacune, ni même un abandon intellectuel en rase campagne face aux armées de l'économie standard, en effet hégémoniques dans l'espace académique international. Au contraire, cette ouverture fait le pari d'une contribution propre des sciences sociales, tant à la connaissance qu'à la critique, pleinement politique, des différentes formes d'oppression sociale et de ravage écologique.

La position exprimée par les économistes Douai et Plumecocq semble a contrario refermer le débat, au moment même où ils entendent l'ouvrir aux autres disciplines. Ils reprochent à certains courants de la socioéconomie écologique de relancer la critique de la réduction économiciste de la valeur, une réduction que partageraient (quoique différemment) l'approche standard et la perspective marxiste. Les auteurs répondent à cette critique en donnant une définition définitive de la valeur qui s'appuie explicitement sur les postulats et la terminologie de Marx: «la valeur économique est une qualité essentiellement inhérente aux marchandises produites par le travail humain et [...] toutes les autres choses du monde (les choses produites pour soi-même, l'air et les autres éléments naturels) ont une valeur d'usage non pas économique, mais qui relève d'autres registres »(p.99). À l'appui de leur axiome, ils citent la position de Jean-Marie 
Harribey: «si on fait le choix de préserver les équilibres des écosystèmes, c'est au nom de valeurs qui ne ressortissent pas à l'économique, mais à l'éthique et au politique " (Harribey, 2015, cité par Douai et Plumecocq, 2017, p. 100). Ainsi conçue, la socioéconomie écologique partirait d'une division claire et étanche entre ce qui est du ressort de l'économique (renvoyant au travail humain, dont la science économique propose la véritable compréhension) et ce qui y échappe (et qui est du ressort du débat social et politique, pluraliste, étudié par d'autres sciences humaines). Ce faisant, le postulat épistémologique de l'exceptionnalité de la sphère économique, envisagée comme le lieu où se produit la vérité du social, est tacitement reconduit. Des apports décisifs postérieurs à Marx passent par la même occasion aux oubliettes, en particulier celui de Karl Polanyi, qui a porté l'attention sur la contribution (sous-estimée par Marx) de l'économie politique comme manière de penser à la "grande transformation» des derniers siècles. Polanyi n'a-t-il pas magistralement montré l'ampleur du travail de «vivisection» qui a été nécessaire pour faire exister une sphère économique distincte du «corps de la société»? Ces actes de vivisection n'ont-ils pas été exécutés par «des gens armés de certitudes que seule la science peut donner» (Polanyi 1983)? N'a-t-il pas fait comprendre, du même coup, que ce ravage peut aussi être saisi comme un "effet de théorie» rendu possible par la concentration d'un «pouvoir symbolique » (Bourdieu, 1984) qui a permis de produire, dans la réalité pratique et sur le corps même de la société, les catégories du savoir théorique qui l'autorise?

\section{Dénoncer le pacte de Parsons}

Cette ligne de partage a priori entre l'économique et le sociopolitique reproduit, on le voit, une opposition entre «la valeur» et «les valeurs». Or, il nous semble précisément qu'une condition de possibilité d'une socioéconomie écologique pluraliste et dynamique consiste à sortir du partage naturalisé et intellectuellement ruineux entre, d'une part, le domaine de la valeur économique (passible d'une élucidation scientifique) et, d'autre part, celui des autres valeurs (culturelles, sociales et morales), variables et changeantes par définition. Depuis des décennies, les relations entre sciences sociales et sciences économiques sont, en effet, davantage caractérisées par une coexistence polie, plus ou moins pacifique, que par une réelle coopération. Pour une raison bien simple: chacun des domaines étudie ce qui vaut à partir d'une division du travail déterminée $a$ priori, selon une sorte d'accord de Yalta.
Cet accord n'est d'ailleurs pas seulement implicite. Comme l'a écrit le sociologue David Stark (2009), il aurait été explicité dès la fin des années 1930, dans l'espace académique états-unien. À l'époque, Talcott Parsons était le patron de la sociologie à l'Université Harvard et publiait un livre fondateur qui définissait la démarche sociologique à suivre, à partir de l'apport des classiques européens (Parsons, 1937). Il aurait alors passé un pacte avec ses collègues économistes afin de les assurer de l'absence d'ambition hégémonique de la nouvelle science sociale sur les questions économiques. Le pacte aurait été énoncé dans les termes suivants: «Vous, vous étudierez la valeur; nous, les sociologues, nous étudierons les valeurs » (nous traduisons, Stark, 2009, p. 7). Pris dans un sens élargi qui dépasse cette anecdote de l'histoire des sciences, le «pacte de Parsons » semble bien avoir été le principe de relations interdisciplinaires qui relève moins de la coopération que de la realpolitik de guerre froide. Le maintien jusqu'à aujourd'hui de ce Yalta disciplinaire est désastreux. Il conduit à faire l'impasse sur des résultats importants produits ces dernières décennies dans le champ de la socioéconomie, qui visent à réinscrire la valeur économique dans un spectre plus vaste. Ces approches ont en commun de rouvrir l'opposition implicitement reconduite et acceptée par les sciences modernes: l'opposition fait/valeur, absolutisée par l'épistémologie promue par les logiciens du cercle de Vienne, et renvoyant plus fondamentalement aux doublets conceptuels objectif/subjectif et universel/particulier (Putnam, 2003 ; Shapin, 2012).

S'inscrivant dans une perspective qui n'est pas sans affinité avec le pragmatisme de William James et John Dewey (Dewey, 2011), de nombreux chercheurs ont, depuis les années 1980, refusé de reproduire ces dualismes en montrant la solidarité matérielle et conceptuelle entre les dynamiques d'évaluation et la production de connaissances sur le monde, à partir d'investigations sur les coordinations sociales empiriquement observables, dont les coordinations économiques ne constituent qu'une partie (Lamont, 2012). Plus précisément, le courant de «l'économie des conventions» a proposé d'inverser la relation de dépendance entre connaissance factuelle et évaluation, en montrant que « la pertinence d'un format de connaissance dépend d'une forme d'évaluation » (Eymard-Duvernay et al., 2006, p.29). Dans cette approche, en effet, «les conventions canalisent l'incertitude à partir d'une forme commune d'évaluation qui qualifie les objets pour la coordination»" (ibid.). L'étude des dynamiques sociales et culturelles d'évaluation (et de leurs appuis matériels et conceptuels) devient alors l'objet privilégié de l'analyse des coordinations humaines. 
Ce déplacement de perspective est riche de conséquences méthodologiques. Les grandeurs économiques, présentées comme objectives et axiologiquement neutres par les pouvoirs publics ou les entreprises, découlent en réalité de partis pris politiques et/ou moraux qu'il est impossible de prétendre expurger, même quand ces grandeurs se présentent sous la forme de tableaux statistiques. Les principes ordinaires qui ordonnent la légitimité d'un ordre économique prétendument «hors social» sont eux-mêmes travaillés par ces partis pris. Le travail fondateur de Luc Boltanski et Laurent Thévenot (1991) a précisément montré combien l'efficacité industrielle ou la légitimité du commerce sont loin d'être des grandeurs «objectives», mais qu'elles sont de part en part traversées par des constructions morales et politiques, qu'illustrent respectivement l'industrialisme de Saint-Simon et l'anthropologie de la relation marchande proposée par Adam Smith. À la suite de ces travaux et de l'apport décisif des science studies, de nombreux chercheurs ont désobéi au pacte de Parsons, en montrant comment la vie économique (les marchés, la monnaie, les marchandises, etc.) est sans cesse animée par des pratiques d'attribution de valeur sociale, mais aussi par des mouvements critiques (Zelizer, 2005). Peu à peu, un espace de recherche a émergé : la sociologie d'attribution de valeur, parfois désignée comme «sociology of worth» (Beckert et Aspers, 2011) ou comme «sociologie de la valuation et de l'évaluation» (Lamont, 2012). Ces travaux ont été particulièrement fructueux dans le domaine de l'étude des controverses écologiques et des dommages environnementaux (Blok, 2011; Centemeri, 2017). Ainsi, Marion Fourcade (2011) a étudié les façons plurielles dont une valeur monétaire était attribuée à ce qui a priori ne pouvait pas en avoir, à savoir les ravages causés par des marées noires.

On se méprend sur la démarche qui anime les tenants d'une approche pluraliste de la socioéconomie écologique en la résumant à une simple visée d'amélioration des indicateurs quantitatifs de la valeur économique en étendant le spectre de ce qui est pris en compte pour construire ces agrégats (comme le bien-être, les services écosystémiques, etc.). On se trompe aussi en identifiant leur pluralisme à une confiance inconsidérée pour les procédures de délibération, qui serait idéalisée comme «instrument de commensurabilité des points de vue divergents pour obtenir une décision légitime» (p. 100). Cette approche, toute empirique, cherche en effet d'abord à saisir comment les acteurs sociaux œuvrent à prendre en compte «ce à quoi ils tiennent» et à distinguer des façons distinctes de les mettre en commun. Ces façons sont inégalement aptes à pouvoir s'inscrire dans les formes requises pour un débat public, fût-il participatif (Thévenot, 2006; Centemeri, 2017). Ces manières ne se laissent pas déduire d'une théorie générale de la valeur, élaborée par le savant, qui s'appliquerait de façon transhistorique. Elles ont besoin du travail empirique des sciences sociales pour être saisies, décrites et rapportées à des modalités identifiées. Elles sont en effet étroitement dépendantes d' «arts de faire ensemble» qui sont inscrits dans des environnements non interchangeables : des réseaux matériels et des équipements culturels qui permettent d'évaluer et de faire émerger des modes d'évaluation les plus ajustés au contexte. Un groupe de victimes mobilisées exprimera ainsi différemment, pour lui-même ou pour le public, la valeur d'un dommage subi, par exemple, un lieu personnellement investi qui a été irrémédiablement perdu suite à un accident industriel, comme la ville lombarde de Seveso en 1976 (Centemeri, 2011).

Il semble donc qu'un sage principe de méthode, en socioéconomie écologique, consiste à ne pas donner $a$ priori une définition déterminée de la valeur, que ce soit celle des classiques, des néoclassiques... ou celle de Marx, dès lors que l'on considère que l'enjeu premier est celui de l'aptitude de nos équipements scientifiques à suivre, au plus proche, les dynamiques de composition de « ce qui vaut» pour les acteurs et les groupes d'acteurs en présence, et les possibilités de transport, vers d'autres espaces, de ces attributions de valeur. Cette approche fondée sur un pluralisme ontologique de la valeur conduit-elle, comme le craignent nos auteurs, à un risque de dépolitisation des enjeux environnementaux? Non. Une telle socioéconomie écologique peut aider à mieux sensibiliser les activistes et les institutions publiques à la pluralité intrinsèque des régimes d'évaluation susceptibles d' ' équiper» les contestations contemporaines des oppressions socioécologiques, en particulier en rendant compte précisément des appuis argumentatifs et des médiations techniques et matérielles grâce auxquels une critique politique peut s'énoncer, être entendue, voire partagée (Thévenot, 2002).

Le précieux dévoilement marxien du «fétichisme de la marchandise» fait partie de cet équipement critique contemporain. Mais il n'en constitue qu'un registre qui ne saurait valoir dans toute situation. Si on lit les travaux portant sur les formes prises empiriquement par les mobilisations contemporaines, on remarque que ce registre marxien ne cesse de cohabiter avec d'autres registres critiques qui se sont développés depuis un siècle et qui ne lui sont pas réductibles: l'écologie politique (Deléage et Hemery, 1989), l'environnementalisme (Ollitrault et Villalba, 2012), la critique écoféministe (Hache, 2016; Salleh, 2017), le souci des communs (Ostrom, 2010; Gutwirth et Stengers, 2016), la critique de la société de surconsommation (Hamilton et Deniss, 2005), la critique du développementalisme occidental portée par des mouvements populaires des pays du Sud, valorisant notamment la survie des cultures autochtones (Kothari et al., 2014 ; Martinez-Alier, 2014)... 
L'ouverture de l'attention à cette pluralité conduit par conséquent à donner une place à des approches qui conçoivent que les rapports des humains avec «la nature » peuvent être saisis de façons qui s'avèrent irréductibles à la perspective marxienne qui, elle, entend ne les saisir qu'à partir du moment où les entités naturelles sont inscrites dans le conflit entre les sociétés humaines et les institutions de capitalisme. Ainsi, les enjeux politiques portés par des mobilisations socioécologiques ne s'expriment pas nécessairement par le langage de la justice sociale, ni même par celui de la liberté ou la dignité des travailleurs. La politisation peut ainsi prendre la forme de la défense d'entités naturelles avec lesquelles les sociétés coexistent (Descola 2005; Escobar 2018). Citons le cas de la lutte du peuple Q'eros, au Pérou, engagé contre les stratégies extractivistes de l'industrie minière dans «leur» montagne, exposée par l'anthropologue Geremia Cometti (2015). Ce rapport de dépendance des sociétés envers le milieu naturel n'est pas la marque des sociétés traditionnelles ou "prémodernes». La crise climatique globale rappelle aujourd'hui aux habitants des mégapoles combien ils dépendent d'entités invisibles (gaz, micro-organismes, etc.) qui font de certains coins de cette Terre des lieux humainement habitables (Latour, 2017).

Ainsi, tant d'un point de vue critique que scientifique, la restriction a priori de ce qui possède une valeur semble conduire à une réduction dommageable de la portée, tant scientifique que politique, de la socioéconomie écologique. Bien avant d'être un concept d'économie politique, « valeur » est une catégorie des langues naturelles renvoyant à ce qui touche la perpétuation de la vie, individuelle ou collective. Les cultures humaines sont de part en part axiologiques, comme les fondateurs de la sociologie l'ont noté (Durkheim, Weber, Simmel) : attribuer une valeur, évaluer, etc., constituent des pratiques culturelles élémentaires, avant d'être des opérations savantes. $\mathrm{La}$ socioéconomie écologique gagne à aviver cet héritage anthropologique qui peut l'aider dans son projet. S'ouvrir aux modalités d'attributions sociales de valeur à la nature lui permet notamment d'ouvrir la critique écologique à la pluralité des trajectoires de résistance au ravage des « communs », en particulier à celui de l'environnement et des cultures vernaculaires généré par le capitalisme. Cela lui permet aussi de prendre en compte le ravage qui est l'œuvre de la violence d'État, parfois justifiée par des motifs anticapitalistes (Gutwirth et Stengers, 2016).

\section{Un point de départ alternatif : la pluralité des façons d'attribuer une valeur à l'environnement}

Douai et Plumecocq considèrent que la distinction marxienne entre valeur et richesse, remarquablement réélaborée par Harribey (2015), permet de poser les bases d'une socioéconomie écologique qui soit à la fois épistémologiquement sûre et clarifiée sur le plan terminologique. Selon Harribey, «la nature n'a pas de valeur intrinsèque » puisqu'elle est du ressort d'un autre ordre, plus large, celui des « richesses » qui sont reconnues comme telles par des opérations éthiques et politiques. $A$ contrario, le domaine de «la valeur économique » reste « une qualité essentiellement inhérente aux marchandises produites par le travail humain » (Harribey, 2015, cité dans Douai et Plumecocq, 2017, p. 99); il est pour eux absurde de mêler les ressources naturelles à des considérations sur la valeur économique, puisque ces dernières «n'acquièrent elles-mêmes de valeur économique que par l'intervention du travail humain » (ibid.,p. 100). Cette pétition de principe explique que la position de l'économiste Joan Martinez-Alier leur paraît « intenable» : ce dernier entend en effet remettre en question la centralité de l'évaluation monétaire de l'environnement et, dans le même temps, «attribuer une valeur économique aux ressources naturelles» (p. 99).

Nous souhaitons ici défendre la cohérence et l'intérêt de la proposition de Martinez-Alier, exposée à de nombreuses reprises, seul ou en collaboration (Martinez-Alier et al., 1998; Martinez-Alier, 2014). Malgré les flottements conceptuels de certaines formulations, son propos nous semble net. Dans ses importants travaux consacrés aux mobilisations relevant de ce qu'il nomme "l'environnementalisme des pauvres ", il entend en effet abandonner le monisme caractérisant l'ensemble des théories économiques de la valeur (néoclassique, keynésienne mais aussi marxienne) pour ce qu'il appelle un pluralisme des «langages de valuation» découlant du refus du monolinguisme promu par l'utilitarisme. L'utilitarisme considère que les valeurs peuvent être traduites sans perte par les grandeurs monétaires: utilité et monnaie sont les médiums de la commensurabilité de l'ordre économique. En s'appuyant sur les travaux d'Otto Neurath (Uebel, 2005), Martinez-Alier a affirmé que la socioéconomie écologique doit faire face à une incomparabilité constitutive. Par conséquent, elle doit faire sienne l'idée, exposée par Kapp, selon laquelle "la tentative de mesurer les coûts sociaux et les bénéfices sociaux simplement en termes de valeur monétaire ou marchande est vouée à l'échec. Ils sont transmis et supportés par la société» (Kapp 1983, cité par Martinez-Alier et al., 1998, nous traduisons). D'un autre côté, la socioéconomie écologique ne peut que prendre acte du fait que la mesure monétaire, mais aussi la mesure énergétique, constituent de puissantes langues véhiculaires, assises sur des réseaux de communication qui leur permettent de circuler plus aisément que d'autres qui sont, elles, 
plus étroitement dépendantes des lieux ou d'idiomes singuliers. En effet, à côté de ces langues quasi universelles qui s'appuient sur des sciences, des métrologies et des institutions, une socioéconomie soucieuse d'un pluralisme des «langages de valuation » doit faire une place aux idiomes qui expriment des constructions culturelles locales, en articulant les relations entre les sociétés humaines et leurs milieux naturels et matériels (Martinez-Alier, 2014). Pour Martinez-Alier, ces idiomes locaux s'expriment de façon privilégiée à l'occasion des conflits environnementaux qui mettent en jeu les communautés humaines dont l'ancrage territorial et la subsistance sont menacés. C'est en particulier le cas dans les pays du Sud, où des communautés d'habitants luttent contre la construction de barrages hydroélectriques, contre des stratégies de déforestation de la forêt pluviale ou contre la destruction de lieux naturels culturellement investis.

Certes, la proposition de Martinez-Alier n'est pas toujours clairement exposée. On peut également discuter la consistance méthodologique de son approche et sa confiance, peut-être naïve, dans la fidélité du tableau de la réalité offert par une pluralité d'indicateurs quantifiés ${ }^{2}$. On peut aussi discuter de la place centrale donnée au métalangage énergétique dans la perspective de MartinezAlier, tendant parfois à occuper la position de dernière instance explicative. Mais le mouvement d'ensemble de sa proposition semble intéressant : celle-ci est en effet l'une des rares à revendiquer une démarche pluraliste, soucieuse de faire droit à une diversité incommensurable de façons d'attribuer une valeur aux milieux dont les sociétés dépendent. C'est pourquoi elle est une contribution importante pour la constitution d'une socioéconomie écologique vraiment interdisciplinaire, dont le geste retrouve, par un autre chemin, le pluralisme développé par le courant conventionnaliste. Ces deux approches partagent une même attention pour l'étude patiente des désaccords, voire des conflits d'interprétation, sur ce qui importe et vaut, pour une société. Leur hybridation offre en outre de bonnes garanties pour une pertinence politique de ce domaine de recherche, dans la mesure où elle permet de rendre compte plus précisément de ce à quoi les mobilisations accordent en pratique une importance dans les conflits environnementaux, à Notre-Dame-des-Landes, à Bure ou à Bhopal. À côté des travaux sociologiques cités plus haut, une socioéconomie écologique plus

\footnotetext{
${ }^{2}$ Nous avons synthétisé et discuté la position de MartinezAlier dans un texte coécrit avec Laura Centemeri (Centemeri et Renou, 2017). J'en profite pour remercier Laura Centemeri pour nos longues conversations dans le cadre du projet Symbios, financé par l'ANR.
}

«polyphonique» gagnera aussi à s'appuyer sur les stimulantes propositions des anthropologues qui s'adressent explicitement à elle, en articulant des analyses qui entrent en écho avec celle ici promue (Graeber, 2001; Tsing, 2017).

En s'inscrivant plus résolument dans l'espace pluraliste des sciences sociales, la socioéconomie écologique trouvera un terrain intellectuel plus fertile, plus riche aussi sur le plan de l'écologie des idées. Ce mouvement impose néanmoins une contrepartie aux participants: la modification d'habitudes académiques héritées du geste modernisateur des sciences de la nature, geste que les science studies ont bien caractérisé (Stengers, 1994). Comme celles des autres univers de la recherche scientifique (Kuhn, 1983), les connaissances générées par des sciences sociales sont étroitement dépendantes de cadres d'évaluation hérités, sans lesquels elles seraient dépourvues de pertinence. Ceux-ci sont des produits de l'histoire, de la technique et de la culture; mais cela ne les empêche pas, au contraire, d'être des outils pertinents, capables de saisir, sous certains aspects, les réalités du monde et d'agir sur elles (Thévenot, 2002). Pour que le mot se transforme en pratique non violente, l' «interdisciplinarité » requise par la socioéconomie écologique demande aux contributeurs qui se retrouvent dans son agenda de recherche de reconnaître le caractère partiel de la portion de réalité rendue visible par la tradition savante dans laquelle ils s'inscrivent. Cette reconnaissance ne saurait être assimilable à la profession de foi d'un relativisme épistémologique : c'est une condition pratique d'une coopération symétrique des savoirs.

Pour répondre aux graves enjeux sociaux et environnementaux des années à venir et pour renforcer la pertinence spécifique des perspectives qu'elle propose, la socioéconomie écologique gagne donc à inscrire le lieu de ses échanges dans un espace intellectuel qui demande aux économistes (de toutes obédiences) et aux autres praticiens d'accepter la légitimité intellectuelle de perspectives sur l'environnement autres que celles sur lesquelles leurs traditions scientifiques se sont constituées. La place des entités naturelles n'y saurait par conséquent être réduite a priori aux seuls statuts de «ressources», de «services» (même «écosystémiques»), voire de «richesses» ayant une «valeur d'usage », dans des interactions envisagées sous l'angle privilégié de leur contribution à la «production». Accepter ce pas de côté - que l'on peut qualifier d'anthropologique - nous semble important, sans quoi on risque de répéter ad absurdum les dialogues de sourds entre la science économique, les sciences sociales et les diverses sensibilités de l'environnementalisme et de l'écologie politique. Il n'est pas certain que les urgences écologiques nous laissent encore ce luxe. 


\section{Références}

Beckert J., Aspers P. (Eds), 2011. The worth of goods. Valuation and pricing in the economy, Oxford, Oxford University Press.

Blanc G., Demeulenaere E., Feuerhahn W. (Eds), 2017. Humanités environnementales. Enquêtes et contre-enquêtes, Paris, Éditions de la Sorbonne.

Blok A., 2011. War of the whales: post-sovereign science and agonistic cosmopolitics in Japanese-global whaling assemblages, Science, Technology and Human Values, 36, 1, 55-81.

Boisvert V., 2017. Économie de l'environnement ou économie écologique, in Blanc G., Demeulenaere E., Feuerhahn W. (Eds), Humanités environnementales. Enquêtes et contre-enquêtes, Paris, Éditions de la Sorbonne, 201-229.

Boltanski L., Thévenot L., 1991. De la justification. Les économies de la grandeur, Paris, Gallimard.

Bourdieu P., 1984. Espace social et genèse des classes, Actes de la recherche en sciences sociales, 52-53, 3-14.

Centemeri L., 2011. Retour à Seveso. La complexité morale et politique du dommage à l'environnement, Annales. Histoire, Sciences Sociales, 66, 1, 213-224.

Centemeri L., 2017. From public participation to place-based resistance. Environmental critique and modes of valuation in the struggles against the expansion of the Malpensa airport, Historical Social Research/Historische Sozialforschung, 42, 3, 97-122.

Centemeri L., Renou G., 2017. Jusqu'où l'économie écologique pense-t-elle les inégalités environnementales? Autour de l'œuvre de Joan Martinez-Alier, in Larrère C. (Ed.), Les inégalités environnementales, Paris, PUF.

Choné A., Hajek I., Hamman P. (Eds), 2016. Guide des humanités environnementales, Villeneuve-d'Ascq, Presses universitaires du Septentrion.

Cometti G., 2015. Lorsque le brouillard a cessé de nous écouter. Changement climatique et migrations chez les Q'eros des Andes péruviennes, Berne, Peter Lang.

Deléage J.-P., Hemery D., 1989. L'écologie, critique de l'économie, L'Homme et la Société, 91-92, 73-86.

Dewey J., 2011. La formation des valeurs, Paris, La Découverte.

Douai A., Plumecocq G., 2017. L'économie écologique, Paris, La Découverte.

Escobar A., 2018. Sentir-penser avec la terre. L'écologie audelà de l'Occident, Paris, Le Seuil.

Eymard-Duvernay F., Favereau O., Orléan A., Salais R., Thévenot L., 2006. Valeurs, coordination et rationalité : trois thèmes mis en relation par l'économie des conventions, Eymard-Duvernay F. (Ed.), L'économie des conventions. Tome 1: Débats, Paris, La Découverte, 23-44.

Foster J.B., Clark B., York R., 2010. The ecological rift. Capitalism's war on the Earth, New York, Monthly Review Press.

Fourcade M., 2011. Cents and sensibility: economic valuation and the nature of "Nature", American Journal of Sociology, $116,6,1721-1777$.
Froger G., Calvo-Mendieta I., Petit O., Vivien F.-D., 2016. Qu'estce que l'économie écologique?, L'économie politique, 79, 8-23.

Graeber D., 2001. Toward an anthropological theory of value. The false coin of our dreams, Palgrave.

Gutwirth S., Stengers I., 2016. Théorie du droit. Le droit à l'épreuve de la résurgence des commons, Revue juridique de l'environnement, 41, 306-343.

Hache E. (Ed.), 2016. Reclaim. Recueil de textes écoféministes, Paris, Cambourakis.

Hamilton C., Deniss R., 2005. Affluenza. When too much is never enough, Crows Nest, Allen \& Unwin.

Harribey J.-M., 2015. La richesse, la valeur et l'inestimable. Fondements d'une critique socioécologique de l'économie capitaliste, Paris, Les liens qui libèrent.

Kothari A., Demaria F., Acosta A., 2014. Buen Vivir, degrowth and ecological Swaraj: alternatives to sustainable development and the green economy, Development, 57, 3-4, 362-375.

Kuhn T.S., 1983. La structure des révolutions scientifiques, Paris, Flammarion.

Lamont M., 2012. Towards a comparative sociology of valuation and evaluation, Annual Review of Sociology, 38, $1,201-221$.

Latour B., 2017. Où atterrir?, Paris, La Découverte.

Martinez-Alier J., Munda G., O’Neill J., 1998. Weak comparability of values as a foundation for ecological economics, Ecological Economics, 26, 3, 277-286.

Martinez-Alier J., 2014. L'écologisme des pauvres. Une étude des conflits environnementaux dans le monde, Paris, Les Petits Matins / Institut Veblen.

Ostrom E., 2010. La gouvernance des biens communs. Pour une nouvelle approche des ressources naturelles, Louvainla-Neuve, De Boeck.

Ollitrault S., Villalba B., 2012. Sous les pavés, la Terre. Mobilisations environnementales en France (1960-2011), entre contestations et expertises, in Pigenet M., Tatarkowksy D. (Eds.), Histoire des mouvements sociaux en France de 1814 à nos jours, Paris, La Découverte, 716-723.

Parsons T., 1937. The structure of social action, New York, McGraw-Hill.

Polanyi K., 1983. La grande transformation, Paris, Gallimard.

Putnam H., 2003. Fait/valeur: la fin d'un dogme, Paris, Éditions de l'éclat.

Salleh A., 2017. Ecofeminism as politics. Nature, Marx and the postmodern, London, Zed Books.

Shapin S., 2012. The sciences of subjectivity, Social Studies of Science, 42, 2, 170-184.

Spash C., 2011. Social ecological economics, American Journal of Economics and Sociology, 72, 2, 340-375.

Stark D., 2009. The sense of dissonance. Accounts of worth in economic life, Princeton, Princeton University Press.

Stengers I., 1994. L'invention des sciences modernes, Paris, La Découverte.

Tanuro D., 2010. L'impossible capitalisme vert, Paris, La Découverte.

Thévenot L., 2002. Which road to follow? The moral complexity of an equipped humanity, in Law J., Mol A. 
(Eds), Complexities. Social studies of knowledge practice, Durham, Duke University Press.

Thévenot L., 2006. L'action au pluriel. Sociologie des régimes d'engagement, Paris, La Découverte.

Tsing A., 2017. Le champignon de la fin du monde, Paris, La Découverte.
Uebel T.S., 2005. Incommensurability, ecology, and planning: Neurath in the socialist calculation debate, 1919-1928, History of Political Economy, 37, 2, 309-342.

Zelizer V., 2005. La signification sociale de l'argent, Paris, Le Seuil.

Citation de l'article : Renou G., 2018. L'avenir de la socioéconomie écologique. Environnement et valeur au-delà du Yalta disciplinaire. Nat. Sci. Soc. 26, 4, 473-480. 